\title{
Symmetry Conservation and Integrals over Local Charge Densities in Quantum Field Theory
}

\author{
M. Requardt \\ Institut für Theoretische Physik, Universität Göttingen, \\ D-3400 Göttingen, Federal Republic of Germany
}

\begin{abstract}
For conserved local currents $\partial^{\mu} j_{\mu}(x)=0$ in quantum field theory it is shown that an $R$-dependence of $\alpha_{R}\left(x_{0}\right)$ in $j_{0}\left(f_{R}(x) \cdot \alpha_{R}\left(x_{0}\right)\right)$ leads to nicer properties than a fixed $\alpha\left(x_{0}\right)$. The behaviour of $\lim _{R \rightarrow \infty} \| j_{0}\left(f_{R}(\boldsymbol{x}) \cdot \alpha_{R}\left(x_{0}\right) \Omega \|\right.$ is discussed under this aspect.
\end{abstract}

\section{Introduction}

In connection with symmetry transformations space integrals over the time component of divergenceless current densities are often considered in relativistic quantum field theory, more rigorously, one smears the current with appropriate testfunctions (see for instance [1] to [6] and further references there). In the following the Wightman framework will be assumed. Let us assume that the global charge operator $Q$ exists with $Q \Omega=0$, where $\Omega$ is to be the unique vacuum vector, that means the symmetry is conserved, then the question arises, in what sense the sequence of local charge operators $Q_{R}:=j_{0}\left(f_{R}(\boldsymbol{x}) \cdot \alpha\left(x_{0}\right)\right)$ converges to the global one. If the time smearing is kept fixed, there is in general only a weak convergence on a dense set of states (the local or quasilocal ones) $([2,5])$. Furthermore there is no hope to prove weak convergence, even if a massgap is assumed, because of the divergence of $\left\|Q_{R} \Omega\right\|$. Moreover there is a close connection between the behavior of $\left\|Q_{R} \Omega\right\|$ and symmetry conservation. If the existence of $Q$ is not a priori assumed, then $\lim _{R \rightarrow \infty}\left\|Q_{R} \Omega\right\|<\infty$ entails symmetry conservation ([7]). It is well known that the rate of divergence of $\left\|Q_{R} \Omega\right\|$ depends on the sequence of testfunctions $\left\{f_{R}(\boldsymbol{x})\right\}([2,3])$. In [4] it was proved that in general $\left\|Q_{R} \Omega\right\|$ always diverges whatever sequence $\left\{f_{R}\right\}$ has been chosen. But there are important examples where this is not true (for instance tensorcurrents of second rank like the energy-momentum tensor [8]). We have proved a generalisation which gives optimal results and which shows that there are examples with $\left\|Q_{R} \Omega\right\|$ bounded in $R$. The results hold true irrespectively of the existence of a gap.

Let $g(p)$ denote $\int \tilde{G}(p)\left|\tilde{\alpha}\left(p_{0}\right)\right|^{2} d p_{0}$ where $\tilde{G}(p)$ is the Fouriertransform of $G(x-y):=(\Omega \mid j(x) j(y) \Omega)$. 
i) If $g(\boldsymbol{p})>c(\boldsymbol{p})^{\alpha}$ with $\alpha<3$ in a neighbourhood of $\boldsymbol{p}=0$ then there exists no sequence $\left\{f_{R}\right\}$ s.t. $\left\|Q_{R} \Omega\right\|$ keeps bounded in $R$.

ii) For $g(\boldsymbol{p})<c|\boldsymbol{p}|^{\beta}, \beta \geqq 3$, there exists $\left\{f_{R}\right\}$ s.t. $\left\|Q_{R} \Omega\right\|<\infty$.

iii) There is no sequence $\left\{f_{R}\right\}$ s.t. $\int\left|\tilde{f}_{R}(\boldsymbol{p})\right|^{2}|\boldsymbol{p}|^{2} d^{3} \boldsymbol{p}<c \cdot R$ where $\tilde{f}_{R}$ denotes the Fourier transform of $f_{R}$, in other words, the sequence $\left\{f_{R}\right\}, f_{R}(x):=f\left(R^{-1} \cdot(x)\right)$, $f(s)=1$ for $0 \leqq s \leqq 1, f=0$ for $s \geqq 2$, which was chosen in [2], leads to the weakest possible divergence.

The proofs of the above statements rely on a somewhat tricky application of Hölder's inequality [9].

The conditions in i), ii) are well adapted to physics because of the special form of covariant currents. For instance if $j_{0}(x)$ is the 0 -component of a covariant vector current or the $0-0$-component of a covariant symmetric tensor current, we get $g(\boldsymbol{p})=|\boldsymbol{p}|^{k} \int_{0}^{\infty}\left|\tilde{\alpha}\left(\sqrt{s+\boldsymbol{p}^{2}}\right)\right|^{2} \cdot\left(s+\boldsymbol{p}^{2}\right)^{-\frac{1}{2}} \varrho(s) d s$ with $k=2,4$ ([8]). From these examples the $\boldsymbol{p}$-factor arises in iii).

Up to now the timesmearing has been kept fixed. The question is now wether we can get better results if the function $\alpha\left(x_{0}\right)$ depends on $R$, without disturbing the physical interpretation of $Q$ (chosing $\alpha\left(x_{0}\right) R$-dependent was a suggestion of Borchers).

\section{2. $\lim _{R \rightarrow \infty}\left\|Q_{R} \Omega\right\|$ with $R$-Dependend Timesmearing}

The class $\left\{f_{R}\right\}$ is chosen like that in iii). The essential object which appears in connection with symmetries generated by conserved currents is the commutator $\left[j_{0}\left(f_{R} \cdot \alpha_{R}\right), A\right]$, with $A \in \mathscr{R}$ where $\mathscr{R}$ denotes the algebra generated by the fields smeared with testfunctions of compact support. For fixed $\alpha_{R}:=\alpha$ the commutator becomes independent of $R$ for $R \geqq R_{A}$ ([1]). The question is now, wether we can find a class $\left\{\alpha_{R}\left(x_{0}\right)\right\}$, s.t. this still holds together with $\left\|j_{0}\left(f_{R} \cdot \alpha_{R}\right) \Omega\right\| \leqq c<\infty$ for all $R$.

Lemma. If $\alpha_{R}\left(x_{0}\right)$ has its support in $\left\{x_{0}|| x_{0} \mid<\frac{1}{2} \cdot R\right\}$, then there exists an $R_{A}$ for every $A \in \mathscr{R}$ with $\left[j_{0}\left(f_{R} \cdot \alpha_{R}\right), A\right]=\left[j\left(f_{R} \cdot \alpha\right), A\right]$ for some fixed $\alpha$ and $R \geqq R_{A}$.

Proof. With $\beta_{R}\left(x_{0}\right):=\int_{-\infty}^{x_{0}}\left(\alpha_{R}-\alpha\right)\left(x_{0}^{\prime}\right) d x_{0}^{\prime}, \int \alpha_{R} d x_{0}=1$ we have

$$
\left[j_{0}\left(f_{R}\left\{\alpha_{R}-\alpha\right\}\right), A\right]=\left[j_{0}\left(f_{R} \partial_{0} \beta_{R}\right), A\right]=\sum_{i}\left[j_{i}\left(\partial_{i} f_{R} \beta_{R}\right), A\right] .
$$

Because of the special form of the $f_{R}$ and the support property of $\beta_{R}\left(\beta_{R}\right.$ has its support in $\left\{x_{0}|| x_{0} \mid<\frac{1}{2} R\right\}$ for sufficiently large $R$ on account of the assumption $\left.\int \alpha_{R} d x=1\right)$ the support of $\partial_{i} f_{R} \beta_{R}$ is spacelike to the support of the testfunctions of every $A \in \mathscr{R}$ for sufficiently large $R$. Hence we get $\left[j_{0}\left(f_{R} \alpha_{R}\right), A\right]=\left[j_{0}\left(f_{R} \alpha\right), A\right]$ for $R \geqq R_{A}$ and both commutators define the same charge operator $Q$.

From now on we choose a special time smearing

$$
\alpha_{R}\left(x_{0}\right) \in \mathscr{D}, \alpha_{R}\left(x_{0}\right):=2 R^{-1} \alpha\left(2 R^{-1} \cdot x_{0}\right)
$$


the support of $\alpha(s)$ being contained in $\{s|| s \mid<1\}, \int \alpha(s) d s=1$. This implies $\tilde{\alpha}_{R}\left(p_{0}\right)=\tilde{\alpha}\left(\frac{1}{2} R p_{0}\right)$.

Theorem. In case of a mass gap there exists a family $\left\{f_{R} \alpha_{R}\right\}$, s.t. $\left\|Q_{R} \Omega\right\|^{2}$ decreases faster than any inverse power of $R$.

Proof. $f_{R} \alpha_{R}$ are chosen as above. Then we get

$$
\left\|Q_{R} \Omega\right\|^{2}=\int_{\tilde{V}}\left|\tilde{f}_{R}\right|^{2}\left|\tilde{\alpha}_{R}\right|^{2} \tilde{G}(p) d^{4} p \quad \text { with } \quad \tilde{V}:=\left\{\left.p\left|p_{0}>0, p_{0}^{2}-\right| \boldsymbol{p}\right|^{2} \geqq m^{2}\right\} .
$$

This equals $R^{6} \int_{\tilde{\Sigma}}|\tilde{f}(R p)|^{2}\left|\tilde{\alpha}\left(\frac{1}{2} R p_{0}\right)\right|^{2} \tilde{G}(p) d^{4} p \leqq R^{6} \int_{\tilde{V}} C_{n^{\prime}}\left(1+R \sqrt{p_{0}^{2}+p^{2}}\right)^{-n^{\prime}} \tilde{G}(p) d^{4} p$, $n^{\prime} \in \mathbb{N}$ arbitrary. $\stackrel{\tilde{G}}{G}(p)$ is a positive measure in $\mathscr{S}^{\prime}$, therefore there exists a $k \in \mathbb{N}$ with

$$
\int_{\tilde{V}}\left(1+\sqrt{p_{0}^{2}+p^{2}}\right)^{-k} \tilde{G}(p) d^{4} p<\infty .
$$

With $R \geqq 1$ we get

$$
\left\|Q_{R} \Omega\right\|^{2} \leqq c R^{6}(1+m R)^{-n} \int_{\tilde{V}}\left(1+\sqrt{p_{0}^{2}+p^{2}}\right)^{-k} \tilde{G}(p) d^{4} p, n^{\prime}=n+k .
$$

As $n$ can be arbitrarily large, the theorem is proved.

Remark. This is especially a simple proof of the well-known fact ([1]) that a mass gap implies conservation of symmetry.

Especially for a conserved vectorcurrent we can get a stronger result. As already mentioned $g(|\boldsymbol{p}|)=|\boldsymbol{p}|^{2} \int_{0}^{\infty}\left|\tilde{\alpha}\left(\frac{1}{2} R \sqrt{s+\boldsymbol{p}^{2}}\right)\right|^{2}\left(s+\boldsymbol{p}^{2}\right)^{-\frac{1}{2}} \varrho(s) d s$ with $\varrho(s)$ a positive measure with support in $\mathbb{R}_{+}$.

Theorem. If $\int_{0}^{\varepsilon} s^{-1} \varrho(s) d s<\infty$ for some $\varepsilon>0, \varrho(s)$ the measure of the covariant vectorcurrent, then $\lim _{R \rightarrow \infty}\left\|Q_{R} \Omega\right\|^{2}<\infty$ with $\alpha_{R}\left(x_{0}\right)$ chosen as above.

Proof. $\left\|Q_{R} \Omega\right\|^{2} \leqq \int\left|\tilde{f}_{R}\right|^{2}|\boldsymbol{p}|^{2} \int_{0}^{\infty} c_{n}\left(1+R \sqrt{s+\boldsymbol{p}^{2}}\right)^{-n} \cdot\left(s+\boldsymbol{p}^{2}\right)^{-\frac{1}{2}} \cdot \varrho(s) d s d^{3} \boldsymbol{p}$. We can split the integral over $s$ into two parts. Only the term

$$
\int_{0}^{\varepsilon_{+}} c_{n}\left(1+R \sqrt{s+\boldsymbol{p}^{2}}\right)^{-n} \cdot\left(s+\boldsymbol{p}^{2}\right)^{-\frac{1}{2}} \varrho(s) d s
$$

needs further calculation ( $\varepsilon_{+}$denotes the closed interval). The estimation of the other term is carried out like the proof of the theorem above. Chosing $n=1$ we get

$$
\int_{0}^{\varepsilon_{+}} c_{1}\left(1+R \sqrt{s+\boldsymbol{p}^{2}}\right)^{-1} \cdot\left(s+\boldsymbol{p}^{2}\right)^{-\frac{1}{2}} \varrho(s) d s \leqq c_{1} R^{-1} \int_{0}^{\varepsilon_{+}} s^{-1} \varrho(s) d s
$$

and $\lim _{R \rightarrow \infty}\left\|Q_{R} \Omega\right\|^{2} \leqq c \lim _{R \rightarrow \infty} R^{-1} \int\left|\tilde{f}_{R}\right|^{2}|\boldsymbol{p}|^{2} d^{3} \boldsymbol{p}$. But $R^{-1} \int\left|\tilde{f}_{R}\right|^{2}|\boldsymbol{p}|^{2} d^{3} \boldsymbol{p}$ is globally bounded in $R$ for the special choice of $\left\{f_{R}\right\}$. This proves the statement.

Remark. If $\varrho(s):=c \cdot s^{-\varkappa}, 0 \leqq \varkappa<1$, then $\left\|Q_{R} \Omega\right\|^{2}=c \cdot R^{2 \varkappa}$. 
The remark shows that we can not weaken the assumption $\int_{0}^{\varepsilon} s^{-1} \varrho(s) d s<\infty$ to $\int_{0}^{\varepsilon} s^{-x} \varrho(s) d s<\infty, 0<x<1$, because $\varrho(s):=\grave{c} \cdot s^{-\frac{1}{2}(1-x)}$ fulfils this assumption, but the corresponding $\left\|Q_{R} \Omega\right\|^{2}$ is divergent.

We can use the above theorem to give an estimation of the behavior of $G(x-y)=\left(\Omega \mid T_{0 i}(x) T_{0 i}(y) \Omega\right), T_{0 i}, \quad(i=1,2,3)$, the $0-i$-component of a conserved irreducible symmetric covariant tensorcurrent.

$\tilde{G}(p)=p_{0}^{2} p_{i}^{2}\left(\varrho_{1}\left(p^{2}\right)+2 \varrho_{2}\left(p^{2}\right)\right)+\left(p_{0}^{2}-\boldsymbol{p}^{2}\right)\left(\boldsymbol{p}^{2}-p_{i}^{2}\right) \varrho_{2}\left(p^{2}\right)+c p_{0}^{2} p_{i}^{2} \delta\left(p^{2}\right)$ with $\varrho_{i}$ positive measures on $R_{+}([8])$. We get $\tilde{G}(p) \leqq p^{2} p_{0}^{2}\left(\varrho_{1}+3 \varrho_{2}+c \delta\right)$ and the right-hand side fulfils the assumption of the theorem.

The results arrived at in this section allow us to give an answer to the question, whether there is always a suitable class $\left\{f_{R} \alpha_{R}\right\}$ of testfunctions, s.t. $\left\|Q_{R} \Omega\right\|$ is bounded in $R$ for the case the corresponding symmetry is conserved. Symmetry conservation is equivalent to $\lim _{R \rightarrow \infty}\left(\Omega \mid Q_{R} E_{0} A \Omega\right)=0$ for all $A \in \mathscr{R}$, where $E_{0}$ is the projection on the states with mass zero ([5]). For a conserved vectorcurrent this means that the measure $\varrho(s)$ has no $\delta$-contribution in $s=0\left(s:=m^{2}\right)$. This is the case for measures like those cited in the remark above, but nevertheless $\left\|Q_{R} \Omega\right\|$ is divergent for all $\left\{f_{R} \alpha_{R}\right\}$.

It follows that symmetry conservation is not equivalent to $\left\|Q_{R} \Omega\right\|$ bounded in $R$ for some suitable class $\left\{f_{R} \alpha_{R}\right\}$.

\section{Summary}

In this paper we have shown that the use of $R$-dependent testfunctions in the time coordinate leads to nicer properties of $j_{0}\left(f_{R} \cdot \alpha_{R}\right)$ than the use of a fixed time smearing. The class $\left\{\alpha_{R}\right\}$ can be chosen in such a way that the commutator $[Q, A]=\lim _{R \rightarrow \infty}\left[Q_{R}, A\right]$, which is the basic object when considering symmetries and global charges, remains unchanged. Moreover, it has been shown that, if a gap is assumed in the mass spectrum, then there exists a class $\left\{f_{R} \cdot \alpha_{R}\right\}$, s.t. $\left\|j_{0}\left(f_{R} \alpha_{R}\right) \Omega\right\|$ as a function of $R$ converges to zero faster than any inverse power of $R$ for $R \rightarrow \infty$. Boundedness of $\left\|Q_{R} \Omega\right\|$ in $R$ has been proved for a somewhat more general situation. This entails that the proofs of several well-known theorems become simpler, for example $\lim _{R \rightarrow \infty}\left\|Q_{R} \Omega\right\|^{2}=0$ entails that $Q_{R}$ converges in the strong sense towards $Q$ on a dense set of states. If there is no mass gap, symmetry conservation, i.e. $\lim \left(\Omega \mid\left[Q_{R}, A\right] \Omega\right)=0$ for all $A \in \mathscr{R}$, does not necessarily imply the existence of a suitable family of testfunctions $\left\{f_{R} \alpha_{R}\right\}$ with $\left\|Q_{R} \Omega\right\|$ being bounded in $R$.

Acknowledgement. The author would like to thank Professor H. Reeh for many helpful remarks and discussions.

\section{References}

1. Kastler, D., Robinson, D. W., Swieca, J. A.: Commun. math. Phys. 2, 108 (1966)

2. Schroer, B., Stichel, P.: Commun. math. Phys. 3, 258 (1966)

3. Reeh,H.: Fortschr. d. Phys. 16, 687 (1968) 
4. Orzalesi, C.A.: Rev. mod. Phys. 42, 381 (1970)

5. Maison, D.: Nuovo Cimento 11A, 389 (1972)

6. Reeh,H.: Symmetries, currents and infinitesimal generators. Lecture-notes Haifa, Aug. 1971

7. Maison, D., Reeh,H.: Commun. math. Phys. 24, 67 (1971)

8. Maison, D., Reeh, H.: Nuovo Cimento 1A, 78 (1971)

9. Requardt, M.: Diplomarbeit Göttingen

Communicated by R. Haag

Received November 24, 1975, in revised form March 25, 1976 
